\title{
An Active Particle in an Activity "Well"
}

\section{A self-propelled particle trapped in a potential well defined by energy availability, has a unique swimming pattern that comes from hidden currents in the fluid in which it swims.}

\section{By Katherine Wright}

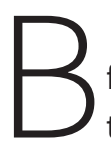
irds, bison, and bacteria all take energy from their environments to move. When these living things - known to physicists as "active" systems-come together in flocks, herds, and swarms, they display behaviors that researchers hope to replicate in reconfigurable materials, for example. But to fully leverage the behaviors, physicists need to understand the fundamental rules that govern idealized versions of these systems. Now Frank Cichos of the University of Leipzig, Germany, and colleagues add another rule to the list of known ones by uncovering how a single active particle behaves when confined in an activity "potential well" $[1,2]$. The team says that their finding could be used to create active ratchets.

In their experiments the team used a gold-coated colloidal particle that "swam" when illuminated with a laser (see Synopsis: Particles Propelled Like Rockets). They placed the particle in a glass-walled, water-filled 2D channel. They then confined its motion by turning the laser off when the particle hit

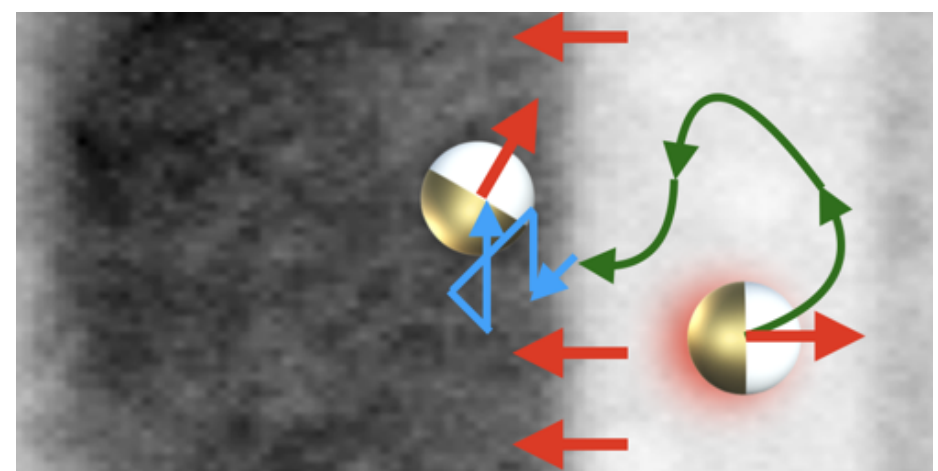

Credit: N. Söker et al. [1] a pre-defined "red line," which acted like the wall of a potential well but applied no force to the particle. They turned the laser on again only when the particle's passive motion took it back inside the well.

The team found that the particle's swimming direction was linked to its position in the well, such that it moved randomly in the well's center but in a preferred direction near the well's red line "wall." The researchers predict that this behavior-which they term polarization-comes from hidden particle-induced currents in the water. Now that they understand how a single active particle moves in an activity well, Cichos says that they plan to explore how multiple particles behave under similar confinement.

Katherine Wright is the Deputy Editor of Physics.

\section{REFERENCES}

1. N. A. Söker et al., "How activity landscapes polarize microswimmers without alignment forces," Phys. Rev. Lett. 126, 228001 (2021).

2. S. Auschra et al., "Polarization-density patterns of active particles in motility gradients," Phys. Rev. E 103, 062601 (2021). 